\title{
Antimicrobial activities of some synthesized macrocyclic pentaazapyridine and dipeptide pyridine derivatives.
}

\author{
Eman M. Flefel ${ }^{1,2 *}$, Mona A. Alsafi' ${ }^{1}$, Sana M. Alahmadi ${ }^{1}$, Abd El-Galil E. Amr ${ }^{3}$, Ahmed A. Fayed ${ }^{2}$ \\ ${ }^{1}$ Department of Chemistry, College of Science, Taibah University, Al-Madinah Al-Monawarah, Saudi Arabia \\ ${ }^{2}$ Department of Photochemistry, Chemical Industries Research Division, National Research Centre, 33 EL-Bohouth St., \\ Dokki, Giza, Egypt \\ ${ }^{3}$ Applied Organic Chemistry Department, National Research Center, Cairo, Dokki, Egypt
}

\begin{abstract}
A series of tetracarboxamide Schiff base and macrocyclic pentaazapyridines has been prepared from 3,5-bis(N-(1-hydrazinyl-1-oxo-3-phenylpropan-2-yl))pyridine carboxamide 4 as starting material, which was synthesized from 3,5-dinicotinic acid 1. Treatment of 4 with 1,4-diaminobutane, 1,6-diaminohexane or cycloalkanone derivatives gave the corresponding macrocyclic tetracarboxamides $5 \mathrm{a}$ and $\mathbf{5 b}$, and cycloalkyl Schiff bases 6a-6c, respectively. Treatment of 4 with acetophenone or acetylpyridine derivatives gave the corresponding Schiff bases 7a-7e and 8a-8c, respectively. Carboxylic acid hydrazide 4 was treated with acid anhydrides in glacial acetic acid to afford the corresponding diimide tetracarboxamides 9-11, respectively. The structures of newly synthesized compounds are established by physical and spectral data evidences. Some of the synthesized compounds were screened as antimicrobial agents.
\end{abstract}

Keywords: 3, 5-Bis (hydrazinyl) pyridine carboxamide, Bis-schiff bases, Macrocyclic pentaazapyridine, Antimicrobial agents.

Accepted on January 06, 2018

\section{Introduction}

The naturally occurring peptide derivatives were identified, and they were used in crucial roles in human physiology, ion channel ligands, including actions as hormones, growth factors, neurotransmitters, or anti-infectives [1-3]. A series of pyridine derivatives are correlated with several pharmacological properties, for example, antimycobacterial [4], anticancer [5], antiviral [6], anti-HIV [7], antifungal and antimicrobial [8], and anticonvulsant [9]. Also, some pyridinecarboxamide analogs were designed and used as PARP-1 inhibitors [10], mycobacterium tuberculosis agents [11] and as CB2 cannabinoid receptor partial agonists [12]. On the other hand, In addition, heterocyclic compounds containing an amino acid or a peptide structural moiety showed biological [13] and antibacterial activities [14]. A branched-chain amino acid (BCAA: Leu, Ile, and Val) mixture has been used for treatment of hypoalbuminemia in patients with decompensated liver cirrhosis in Japan [15]. In view of these observations and in continuation of our previous work [16-24] in heterocyclic and peptide chemistry, we have synthesized some new 3, 5-bis(carboxamide Schiff base) pyridines and macrocyclic pentaazapyridines and we have evaluated some of them as antimicrobial agents.

\section{Results and Discussion}

\section{Chemistry}

In this study, we report a series of linear dipeptide Schiff base derivatives by using N2, N2'-(pyridine-3, 5-dicarbonyl)-di-Lphenyalaninyl hydrazide (4) as starting material, which was synthesized from 3, 5-pyridinedicarboxylic acid according to a reported procedure [4] (Scheme 1).

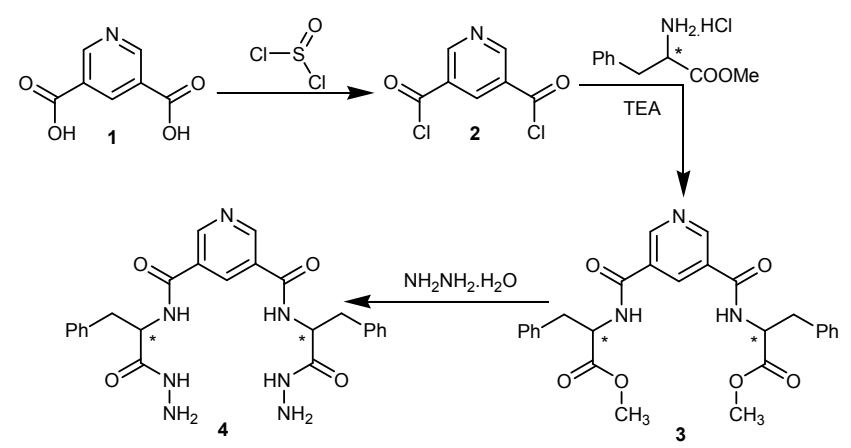

Scheme 1. Synthetic routes to starting compound 4.

Treatment of 3, 5-bis-hydrazide 4 with 1, 4-diaminobutane or 1, 6-diaminohexane afforded the corresponding macrocyclic tetracarboxamide derivatives $\mathbf{5 a}$ and $\mathbf{5 b}$. Condensation of $\mathbf{4}$ 
with cycloalkanones in refluxing glacial acetic acid gave the corresponding cycloalkanyl Schiff base derivatives 6a-6c. Additionally, reaction of $\mathbf{4}$ with acetophenone or acetylpyridines gave the corresponding Schiff base derivatives 7a-7e and 8a-8c, respectively (Scheme 2).

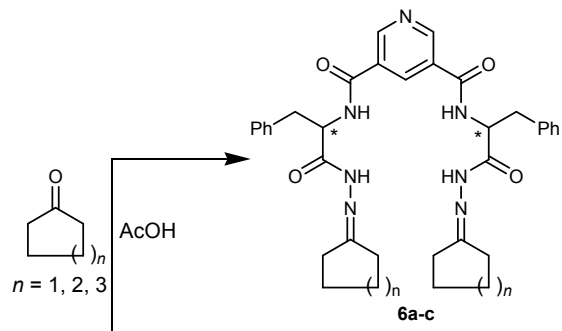

$\mathbf{a}, \mathrm{n}=1 ; \mathbf{b}, \mathrm{n}=2 ; \mathbf{c}, \mathrm{n}=3$
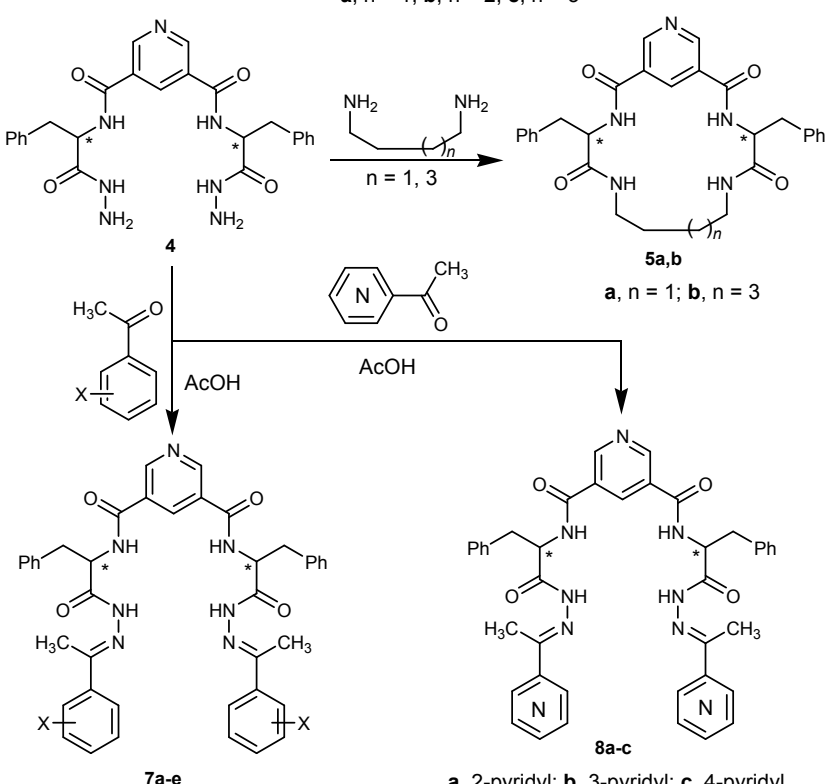

a, $X=H, b, X=M e, c, X=O M e$,
d, $X=C l, e X=F$
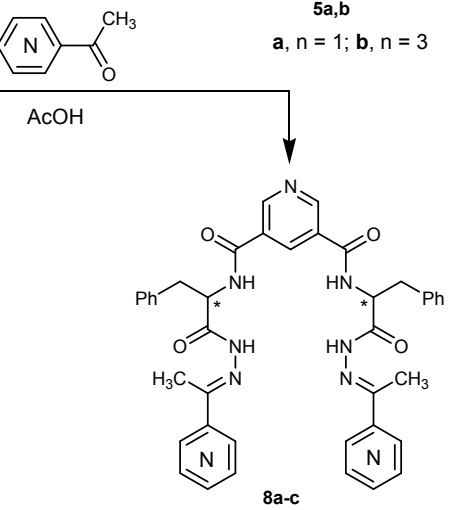

a, 2-pyridyl; b, 3-pyridyl; c, 4-pyridyl

Scheme 2. Synthetic routes to compounds $5 a$ and $5 b, 6 a-6 c, 7 a-7 e$ and $8 a-8 c$.

Finally, carboxylic acid hydrazide 4 was treated with acid anhydrides, namely, phthalic, tetrachloro-phthalic, 1, 8naphthaline or 2, 3-pyridinedicarboxylic acid anhydride in glacial acetic acid to afford the corresponding diimide tetracarboxamide derivatives 9-11, respectively (Scheme 3).

\section{Antimicrobial activity}

The newly synthesized compounds 5-11 were tested for their preliminary antimicrobial activity against different microorganisms representing gram-positive (Staphylococcus aureus, Bacillus aureus and Bacillus subtilis), gram-negative bacteria (Escherichia coli), fungi (Aspergillus niger) and yeast (Candida albican). The obtained results (Table 1) showed that all synthesized compounds exhibited both antibacterial and antifungal activities on all tested microbial strains, except for compounds 5a, 5b, 7a, 7e, 8a, 8c, 9 and 11, which did not showed antifungal activity against Candida albicans. In terms of antifungal activities, compounds $5 \mathbf{a}, \mathbf{7 c}, \mathbf{7 d}$ and $\mathbf{1 0}$ were the most active and their activities was higher than that of the positive control (fusidic acid) by about 2.6, 2.6, 5.0 and 5.0\%, respectively. Regarding antibacterial activities, it can be clearly observed that compounds 6a, 6b, 7d, 8b, 10 and 11 were the highly active compounds.

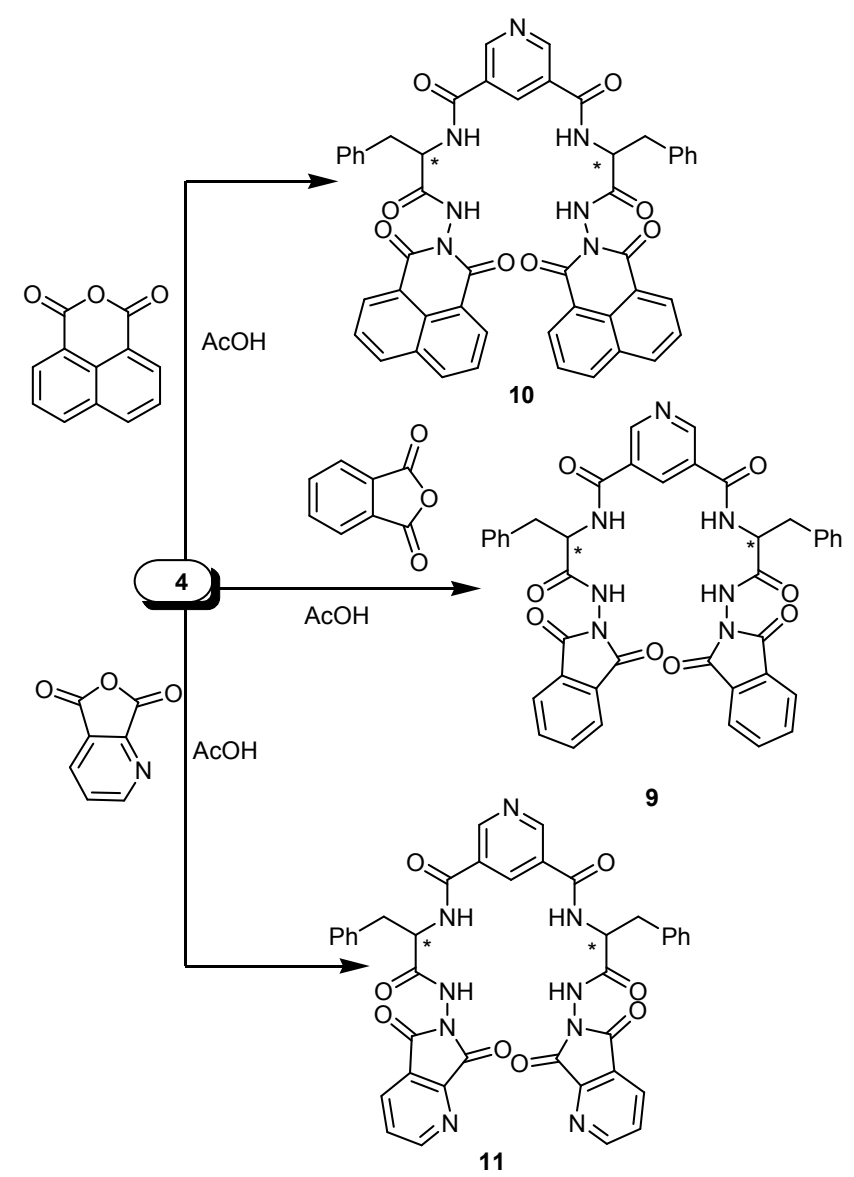

Scheme 3. Synthetic routes to compounds 9-11.

Table 1. Antimicrobial activities of some newly synthesized compounds.

\begin{tabular}{|c|c|c|c|c|c|c|}
\hline \multirow[t]{3}{*}{ Compound } & \multicolumn{6}{|l|}{ Inhibition zome $(\mathrm{cm})$} \\
\hline & \multicolumn{2}{|l|}{ Gram +ve } & \multicolumn{2}{|l|}{ Gram -ve } & \multirow{2}{*}{$\begin{array}{l}\text { Fungi } \\
\text { Aspergillus niger }\end{array}$} & \multirow{2}{*}{$\begin{array}{l}\text { Yeast } \\
\text { Candida albicans }\end{array}$} \\
\hline & Staphylococcus aureus & Bacillus subtilis & Bacillus aureus & Escherichia coli & & \\
\hline $5 a$ & 1.65 & 1.46 & 1.80 & 0.66 & 1.95 & - \\
\hline $5 b$ & 1.68 & 1.75 & 1.55 & 0.60 & 1.70 & - \\
\hline
\end{tabular}




\begin{tabular}{|c|c|c|c|c|c|c|}
\hline $6 \mathbf{a}$ & 1.80 & 1.65 & 1.96 & 0.78 & 1.48 & 0.94 \\
\hline $6 b$ & 1.85 & 1.85 & 1.92 & 0.80 & 1.56 & 0.92 \\
\hline $6 c$ & 1.76 & 1.72 & 1.58 & 0.74 & 1.80 & 0.95 \\
\hline $7 a$ & 1.56 & 1.56 & 1.50 & 0.66 & 1.68 & - \\
\hline $7 b$ & 1.75 & 1.65 & 1.74 & 0.75 & 1.85 & 1.05 \\
\hline $7 c$ & 1.64 & 1.76 & 1.75 & 0.80 & 1.95 & 1.05 \\
\hline $7 d$ & 1.95 & 1.85 & 2.00 & 0.90 & 2.00 & 0.96 \\
\hline $7 e$ & 1.78 & 1.45 & 1.65 & 0.62 & 1.75 & - \\
\hline $8 a$ & 1.55 & 1.85 & 1.50 & 0.65 & 1.75 & - \\
\hline $8 b$ & 1.85 & 1.80 & 1.95 & 0.78 & 1.55 & 1.00 \\
\hline $8 c$ & 1.66 & 1.72 & 1.22 & 0.60 & 1.75 & - \\
\hline 9 & 1.65 & 1.65 & 1.83 & 0.64 & 1.58 & - \\
\hline 10 & 1.77 & 1.84 & 1.88 & 0.92 & 2.00 & 1.10 \\
\hline 11 & 1.80 & 1.70 & 1.65 & 0.64 & 1.75 & - \\
\hline Chloramphenicol & 2.00 & 2.00 & 2.10 & 0.95 & - & - \\
\hline Fusidic acid & - & - & - & - & 1.9 & 1.9 \\
\hline
\end{tabular}

\section{Experimental section}

Chemistry: Melting points were determined in open glass capillary tubes with an Electro Thermal Digital melting point apparatus (model: IA9100) and are uncorrected. Elemental microanalysis for carbon, hydrogen and nitrogen (Microanalytical Unit, NRC) was found within the acceptable limits of the calculated values. IR was recorded on a Nexus 670 FTIR Nicolet, Fourier Transform infrared spectrometer. ${ }^{1} \mathrm{H}$ $\mathrm{N}$ MR and ${ }^{13} \mathrm{C}$ NMR spectra were run in DMSO-d6 on Jeol $500 \mathrm{MHz}\left({ }^{1} \mathrm{H}\right)$ and $125 \mathrm{MHz}\left({ }^{13} \mathrm{C}\right)$ instruments. Mass spectra were run on a MAT Finnigan SSQ 7000 spectrometer, using the Electron Impact technique (EI). Analytical Thin Layer Chromatography (TLC) was performed on silica gel aluminum sheets, 60 F254 (E. Merck). Antimicrobial activities were evaluated in National Research Center, Dokki, Cairo, Egypt.

Synthesis of cyclo-(Na-dinicotinoyl)-bis (Lphenylalaninyl)-1, 4- or 1, 6-alkanediamine 5a and 5b: To a cold $\left(-5^{\circ} \mathrm{C}\right)$ and stirred solution of the dihydrazide $4(1 \mathrm{mmol})$ in $5 \mathrm{~N}$ aq. $\mathrm{HCl}(3 \mathrm{ml})$ and acetic acid $(3 \mathrm{ml})$, sodium nitrite solution $(10 \%, 0.13 \mathrm{~g}, 2 \mathrm{mmol})$ was added at the same temperature. Stirring was continued for $30 \mathrm{~min}$, the reaction mixture was extracted with ether, washed with water, $\mathrm{NaHCO}_{3}$, and water, dried over anhydrous sodium sulfate. The cold ethereal solution $\left(-5^{\circ} \mathrm{C}\right)$ was then added to a cold $\left(-5^{\circ} \mathrm{C}\right)$ dichloromethane solution of 1, 4-butanediamine, or 1, 6hexanediamine $\left(1 \mathrm{mmol}, 10 \mathrm{ml}\right.$ of $\left.\mathrm{CH}_{2} \mathrm{Cl}_{2}\right)$. Stirring was continued for $5 \mathrm{~h}$ at $-5^{\circ} \mathrm{C}$ and at room temperature for $2 \mathrm{~h}$. The reaction mixture was washed with $1 \mathrm{~N}$ hydrochloric acid, water and then dried over anhydrous calcium chloride. The solvent was evaporated under reduced pressure and crystallized from ethanol/ether to afford the corresponding title compounds $\mathbf{5 a}$ and $\mathbf{5 b}$, respectively.

Cyclo-(No-dinicotinoyl)-bis [L-phenylalaninyl]-1, 4butanediamine (5a): Yield, 58\%; M. p. $204-206^{\circ} \mathrm{C}$; $[\alpha]^{25} \mathrm{D}^{=-110}(\mathrm{c}=0.5, \mathrm{DMF}) ; \mathrm{IR}(\mathrm{KBr}) v_{\max }$ in $\mathrm{cm}^{-1}: 3365(\mathrm{NH})$, 3076 (CH-Ar), 2984 (CH-aliph.), 1662, 1535, 1234 (C=O, amides I-III) $\mathrm{cm}^{-1} ;{ }^{1} \mathrm{H}$ MR (DMSO-d6, $\delta$ ppm): $1.40-1.48$ (m, $\left.4 \mathrm{H}, 2 \mathrm{CH}_{2}\right), 2.62-2.74\left(\mathrm{~m}, 4 \mathrm{H}, 2 \mathrm{CH}_{2}\right), 3.42\left(\mathrm{~d}, 4 \mathrm{H}, 2 \mathrm{CH}_{2}\right)$, 4.05-4.10 (m, $2 \mathrm{H}, 2 \mathrm{CH}), 6.95-7.52(\mathrm{~m}, 10 \mathrm{H}, 2 \mathrm{Ph}-\mathrm{H}), 8.58$, $9.05(2 \mathrm{~s}, 3 \mathrm{H}$, pyr-H), 8.72, $8.95(2 \mathrm{~s}, 4 \mathrm{H}, 4 \mathrm{NH}$, exchangeable with $\mathrm{D}_{2} \mathrm{O}$ ); MS, m/z (\%): 514 (12) (M) ${ }^{+}$; Anal. Calcd. for $\mathrm{C}_{29} \mathrm{H}_{31} \mathrm{~N}_{5} \mathrm{O}_{4}$ (513.58): C 67.82, H 6.08, N 13.64; found $\mathrm{C} 67.70, \mathrm{H} 6.00, \mathrm{~N} 13.57$.

Cyclo-(No-dinicotinoyl)-bis[L-phenylalaninyl]-1, $6-$

hexanediamine (5b): Yield, $60 \%$; M. p. $194-196^{\circ} \mathrm{C} ;[\alpha]^{25} \mathrm{D}=-96$ (c=0.5, DMF); IR (KBr) $v_{\max }$ in $\mathrm{cm}^{-1}: 3358-3312(\mathrm{NH}), 3080$ (CH-Ar), 2975 (CH-aliph.), 1660, 1530, 1240 (C=O, amides IIII) $\mathrm{cm}^{-1}$; ${ }^{1} \mathrm{H}$ MR (DMSO-d6, $\left.\delta \mathrm{ppm}\right): 1.24-1.28(\mathrm{~m}, 4 \mathrm{H}, 2$ $\left.\mathrm{CH}_{2}\right), 1.38-1.44\left(\mathrm{~m}, 4 \mathrm{H}, 2 \mathrm{CH}_{2}\right), 2.60-1.68\left(\mathrm{~m}, 4 \mathrm{H}, 2 \mathrm{CH}_{2}\right)$, 3.42 (d, $\left.4 \mathrm{H}, 2 \mathrm{CH}_{2}\right), 4.08-4.20$ (m, $\left.2 \mathrm{H}, 2 \mathrm{CH}\right), 6.96-7.55(\mathrm{~m}$, $10 \mathrm{H}, 2 \mathrm{Ph}-\mathrm{H}), 8.62,9.00$ (2 s, $3 \mathrm{H}$, pyridyl-H), 8.74, 8.95 (42 $\mathrm{s}, \mathrm{H}, 4 \mathrm{NH}$, exchangeable with $\left.\mathrm{D}_{2} \mathrm{O}\right) ; \mathrm{MS}, \mathrm{m} / \mathrm{z}(\%): 542$ (22) (M) ${ }^{+}$; Anal. Calcd. for $\mathrm{C}_{31} \mathrm{H}_{35} \mathrm{~N}_{5} \mathrm{O}_{4}$ (541.64): C 68.74, $\mathrm{H}$ 6.51, N 12.93; found C 68.62, H 6.44, N 12.85 .

Synthesis of Schiff bases 6a-6c: To a solution of acid hydrazide 4 (1 mmol) in glacial acetic acid $(30 \mathrm{ml})$, cyclopentanone, cyclohexanone or cycloheptanone $(2 \mathrm{mmol})$ was added. The reaction mixture was refluxed for $6 \mathrm{~h}$, poured onto ice water, the obtained solid was filtered off, washed with water, dried, and crystallized from the proper solvents to give the corresponding Schiff bases derivatives 6a-6c, respectively. 
$N, N$ '-Bis [1-(cyclopentanyl-1-methylhydrazonyl)-2-L-

phenylalaninyl]-3,5-(diaminocarbonyl) pyridine (6a): Yield, $82 \%$; M. p. $178-180^{\circ} \mathrm{C}\left(\mathrm{EtOH} / \mathrm{H}_{2} \mathrm{O}\right) ;[\alpha]^{25} \mathrm{D}^{=-124}(\mathrm{c}=0.5$, $\mathrm{DMF}$ ); IR (KBr) $v_{\max }$ in $\mathrm{cm}^{-1}: 3446-3367$ (NH), 3085 (CH-Ar), 2982 (CH-aliph.), 1661, 1521, 1314 (C=O, amides I-III) $\mathrm{cm}^{-}{ }^{1}$; ${ }^{1} \mathrm{H}$ MR (DMSO-d6, $\left.\delta \mathrm{ppm}\right): 1.28-1.34(\mathrm{~m}, 16 \mathrm{H}, 2$ cyclopentyl-H), 3.36 (d, $\left.4 \mathrm{H}, 2 \mathrm{CH}_{2}\right), 4.62-4.73(\mathrm{~m}, 2 \mathrm{H}, 2$ $\mathrm{CH}), 6.98-7.52$ (m, $10 \mathrm{H}, 2 \mathrm{Ph}-\mathrm{H}), 7.90,8.64(2 \mathrm{~s}, 4 \mathrm{H}, 4 \mathrm{NH}$, exchangeable with $\left.\mathrm{D}_{2} \mathrm{O}\right), 8.68,9.12\left(2 \mathrm{~s}, 3 \mathrm{H}\right.$, pyr-H); ${ }^{13} \mathrm{C}-$ NMR (DMSO-d6): 41.35 (2 C, $\left.2 \mathrm{CH}_{2}\right), 53.14$ (2 C, $\left.2 \mathrm{CH}\right)$, 125.66, 127.70, 128.64, 139.44 (12 C, 2 Ph-C), 131.46, 141.23, 152.36 (5 C, Pyr-C), 167.18 (2 C, 2 C=O), 176.64 (2 C, 2 $\mathrm{C}=\mathrm{O}), 26.04,36.86,186.74$ (10 C, cyclopentyl-C); MS, m/z (\%): 622 (6) (M) ${ }^{+}$; Anal. Calcd. for $\mathrm{C}_{35} \mathrm{H}_{39} \mathrm{~N}_{7} \mathrm{O}_{4}$ (621.72): $\mathrm{C}$ 67.61, H 6.32, N 15.77; found C 67.52, H 6.22, N 15.69.

$N, N$ '-Bis [1-(cyclohexanyl-1-methylhydrazonyl)-2-L-

phenylalaninyl]-3,5-(diaminocarbonyl)pyridine (6b): Yield, 66 $\%$; M. p. $206-208^{\circ} \mathrm{C}\left(\mathrm{AcOH} / \mathrm{H}_{2} \mathrm{O}\right) ;[\alpha]^{25} \mathrm{D}=-116(\mathrm{c}=0.5$, $\mathrm{DMF})$; IR (KBr) $v_{\max }$ in $\mathrm{cm}^{-1}: 3565-3432(\mathrm{NH}), 3084$ (CH-Ar), 2974 (CH-aliph.), 1664, 1525, 1318 (C=O, amides I-III) $\mathrm{cm}^{-1}$; ${ }^{1} \mathrm{H}$ MR (DMSO-d6, $\delta$ ppm): 1.25-1.36 (m, 20 H, 2 cyclohexyl$\mathrm{H}), 3.42$ (d, $\left.4 \mathrm{H}, 2 \mathrm{CH}_{2}\right), 4.65-4.70(\mathrm{~m}, 2 \mathrm{H}, 2 \mathrm{CH}), 6.96-7.54$ (m, $10 \mathrm{H}, 2 \mathrm{Ph}-\mathrm{H}), 7.96,8.68(2 \mathrm{~s}, 4 \mathrm{H}, 4 \mathrm{NH}$, exchangeable with $\mathrm{D}_{2} \mathrm{O}$ ), 8.76, $9.10(2 \mathrm{~s}, 3 \mathrm{H}, \mathrm{Pyr}-\mathrm{H}) ;{ }^{13} \mathrm{C}-\mathrm{NMR}$ (DMSOd6): 41.36 (2 C, $\left.2 \mathrm{CH}_{2}\right), 53.32(2 \mathrm{C}, 2 \mathrm{CH}), 125.65,127.74$, 128.67, 139.48 (12 C, 2 Ph-C), 131.42, 141.28, 152.33 (5 C, Pyr-C), 167.24 (2 C, 2 C=O), 176.44 (2 C, $2 \mathrm{C}=\mathrm{O}), 23.22$, 26.86, 28.14, 161.33 (12 C, cyclohexyl-C); MS, m/z (\%): 650 (22) (M) ${ }^{+}$; Anal. Calcd. for $\mathrm{C}_{37} \mathrm{H}_{43} \mathrm{~N}_{7} \mathrm{O}_{4}$ (649.78): $\mathrm{C} 68.39, \mathrm{H}$ 6.67, N, 15.09; found C 68.30, H 6.60, N 15.00.

$N, N$ '-Bis [1-(cycloheptanyl-1-methylhydrazonyl)-2-L-

phenylalaninyl]-3,5-(diaminocarbonyl)pyridine (6c): Yield, 72 \%; M. p. $232-234^{\circ} \mathrm{C}(\mathrm{EtOH}) ;[\alpha]^{25} \mathrm{D}^{=-112}$ (c=0.5, DMF); IR $(\mathrm{KBr})$ vmax in $\mathrm{cm}^{-1}$ : 3554-3428 (NH), $3090(\mathrm{CH}-\mathrm{Ar}), 2978$ (CH-aliph.), 1661, 1526, $1318\left(\mathrm{C}=\mathrm{O}\right.$, amides I-III) $\mathrm{cm}^{-1} ;{ }^{1} \mathrm{H}$ MR (DMSO-d6, $\delta$ ppm): 1.20-1.36 (m, 24 H, 2 cycloheptyl-H), $3.31\left(\mathrm{~d}, 4 \mathrm{H}, 2 \mathrm{CH}_{2}\right)$, 4.62-4.71 (m, $\left.2 \mathrm{H}, 2 \mathrm{CH}\right), 6.99-7.56(\mathrm{~m}$, $10 \mathrm{H}, 2 \mathrm{Ph}-\mathrm{H}), 7.94,8.66(2 \mathrm{~s}, 4 \mathrm{H}, 4 \mathrm{NH}$, exchangeable with $\left.\mathrm{D}_{2} \mathrm{O}\right), 8.79,9.18$ (2 s, $\left.3 \mathrm{H}, \mathrm{Pyr}-\mathrm{H}\right) ;{ }^{13} \mathrm{C}-\mathrm{NMR}$ (DMSO-d6): 41.48 ( 2 C, $2 \mathrm{CH}_{2}$ ), 53.15 (2 C, $\left.2 \mathrm{CH}\right), 125.76,127.54,128.62$, 139.50 (12 C, 2 Ph-C), 132.13, $141.18,152.42$ (5 C, Pyr-C), 167.24 ( $2 \mathrm{C}, 2 \mathrm{C}=\mathrm{O}), 175.98$ (2 C, $2 \mathrm{C}=\mathrm{O}), 23.56,25.42$, 29.70, 183.84 (14 C, cycloheptyl-C); MS, m/z (\%): 678 (15) $(\mathrm{M})^{+}$; Anal. Calcd. for $\mathrm{C}_{39} \mathrm{H}_{47} \mathrm{~N}_{7} \mathrm{O}_{4}$ (677.83): C 69.10, H 6.99, N 14.46; found C 69.00, H 6.90, N 14.40.

Synthesis of compounds 7a-7e and 8a-8c: A mixture of 4 (1 mmol) and a substituted acetophenone (acetophenone, 4methyl-, 4-methoxy-, 4-chloro-, 4-fluoroacetophenone) or an acetylpyridine (2-acetyl-, 3-acetyl-, 4-acetylpyridine, $2 \mathrm{mmol}$ ) in glacial acetic acid $(30 \mathrm{ml})$ was refluxed for $4-7 \mathrm{~h}$. The reaction mixture was poured into ice-water, and then neutralized with $1 \mathrm{~N}$ aq. sodium carbonate. The obtained solid was filtered off, washed with water, dried, and crystallized from the proper solvent to give the corresponding Schiff bases 7a-7e and 8a-8c, respectively.
$N, N$ '-Bis [(1-phenyl-1-methyl-hydrazonyl-2-L-

phenylalaninyl]-3,5-(diaminocarbonyl)pyridine (7a): Yield, 75 $\%$; M. p. $242-244^{\circ} \mathrm{C}\left(\mathrm{DMF} / \mathrm{H}_{2} \mathrm{O}\right) ;[\alpha]^{25} \mathrm{D}^{=-98}(\mathrm{c}=0.5, \mathrm{DMF})$; IR (KBr) vmax in $\mathrm{cm}^{-1}$ : 3367-3342 (NH), 3078 (CH-Ar), 2990 (CH-aliph.), 1652, 1537, 1254 (CO, amides I-III) $\mathrm{cm}^{-1} ;{ }^{1} \mathrm{H} \mathrm{MR}$ (DMSO-d6, $\delta$ ppm): 1.04 (s, $\left.6 \mathrm{H}, 2 \mathrm{CH}_{3}\right), 3.24$ (d, $4 \mathrm{H}, 2 \mathrm{CH}_{2}$ ), 4.68-4.75 (m, $2 \mathrm{H}, 2 \mathrm{CH}), 7.05-7.68$ (m, $20 \mathrm{H}, 4 \mathrm{Ph}-\mathrm{H}), 8.54$, $8.65\left(2 \mathrm{~s}, 4 \mathrm{H}, 4 \mathrm{NH}, \mathrm{D}_{2} \mathrm{O}\right.$ exchangeable), 8.75, $9.08(2 \mathrm{~s}, 3 \mathrm{H}$, pyr-H); ${ }^{13} \mathrm{C}-\mathrm{NMR}$ (DMSO-d6): $14.43\left(2 \mathrm{C}, 2 \mathrm{CH}_{3}\right), 41.22$ (2 C, $\left.2 \mathrm{CH}_{2}\right), 53.98(2 \mathrm{C}, 2 \mathrm{CH}), 125.56,127.72,128.55,139.35$ (12 C, 2 Ph-C), 127.82, 128.55, 130.70, 133.36 (12 C, 2 Ph-C), 131.72, 140.34, 151.86 (5 C, pyr-C), 167.42 (2 C, 2 CO), 168.35 ( 2 C, 2 C=N), 176.98 ( 2 C, 2 CO); MS, m/z (\%): 694 (16) (M) ${ }^{+}$. Anal. Calcd. for $\mathrm{C}_{41} \mathrm{H}_{39} \mathrm{~N}_{7} \mathrm{O}_{4}$ (693.79): $\mathrm{C} 70.98, \mathrm{H}$ 5.67, N 14.13; found C 70.90, H 5.60, N 14.05.

N,N'-Bis[1-(4-methylphenyl-1-methylhydrazonyl)-2-L-

phenylalaninyl]-3,5-(diaminocarbonyl)pyridine (7b): Yield, 68 \%; M. p. $226-228^{\circ} \mathrm{C}\left(\mathrm{AcOH} / \mathrm{H}_{2} \mathrm{O}\right) ;[\alpha]^{25} \mathrm{D}=-105$ (c=0.5, DMF); IR (KBr) vmax in $\mathrm{cm}^{-1}$ : 3388-3335 (NH), 3082 (CH-Ar), 2985 (CH-aliph.), 1653, 1534, 1252 (CO, amides I-III) $\mathrm{cm}^{-1} ;{ }^{1} \mathrm{H} \mathrm{MR}$ (DMSO-d6, $\delta \mathrm{ppm}): 1.00\left(\mathrm{~s}, 6 \mathrm{H}, 2 \mathrm{CH}_{3}\right), 2.23\left(\mathrm{~s}, 6 \mathrm{H}, 2 \mathrm{CH}_{3}\right)$, $3.26\left(\mathrm{~d}, 4 \mathrm{H}, 2 \mathrm{CH}_{2}\right), 4.65-4.72(\mathrm{~m}, 2 \mathrm{H}, 2 \mathrm{CH}), 7.12-7.72(\mathrm{~m}$, $18 \mathrm{H}, 4 \mathrm{Ph}-\mathrm{H}), 8.65,8.78$ (2 s, $4 \mathrm{H}, 4 \mathrm{NH}, \mathrm{D}_{2} \mathrm{O}$ exchangeable), 8.72, 9.12 (2 s, $3 \mathrm{H}$, pyr-H); ${ }^{13} \mathrm{C}-\mathrm{NMR}$ (DMSO-d6): 14.22, $23.45\left(4 \mathrm{C}, 4 \mathrm{CH}_{3}\right), 41.25\left(2 \mathrm{C}, 2 \mathrm{CH}_{2}\right), 54.10(2 \mathrm{C}, 2 \mathrm{CH})$, 125.62, 127.70, 128.58, 139.45 (12 C, 2 Ph-C), 128.74, 129.08, 130.86, 140.35 (12 C, 2 Ph-C), 131.84, 140.38, 151.85 (5 C, pyr-C), 167.36 (2 C, 2 CO), 168.45 (2 C, 2 C=N), 176.95 (2 C, $2 \mathrm{CO}) ; \mathrm{MS}, \mathrm{m} / \mathrm{z}(\%)$ : $722(8)(\mathrm{M})^{+}$; Anal. Calcd. for $\mathrm{C}_{43} \mathrm{H}_{43} \mathrm{~N}_{7} \mathrm{O}_{4}$ (721.84): $\mathrm{C} 71.55, \mathrm{H} 6.00, \mathrm{~N} 13.58$; found $\mathrm{C}$ 71.48, H 5.92, N 13.50.

$N, N$ '-Bis [1-(4-methoxylphenyl-1-methylhydrazonyl)-2-Lphenylalaninyl]-3,5-(diaminocarbonyl)pyridine (7c). Yield, 78 $\%$; M. p. $268-270^{\circ} \mathrm{C}$ (dioxane); $[\alpha]^{25} \mathrm{D}^{=-78}$ (c=0.5, DMF); IR (KBr) vmax in $\mathrm{cm}^{-1}$ : 3374-3328 (NH), 3080 (CH-Ar), 2988 (CH-aliph.), 1654, 1535, 1255 (CO, amides I-III) cm ${ }^{-1}$; ${ }^{1} \mathrm{H} \mathrm{MR}$ (DMSO-d6, $\delta$ ppm): 1.12 (s, $\left.6 \mathrm{H}, 2 \mathrm{CH}_{3}\right), 3.32\left(\mathrm{~d}, 4 \mathrm{H}, 2 \mathrm{CH}_{2}\right.$ ), $2.68\left(\mathrm{~s}, 6 \mathrm{H}, 2 \mathrm{OCH}_{3}\right), 4.62-4.68(\mathrm{~m}, 2 \mathrm{H}, 2 \mathrm{CH}), 6.95-7.65(\mathrm{~m}$, $18 \mathrm{H}, 4 \mathrm{Ph}-\mathrm{H}), 8.68,8.82$ (2 s, $4 \mathrm{H}, 4 \mathrm{NH}, \mathrm{D}_{2} \mathrm{O}$ exchangeable), 8.74, 9.10 (2 s, $3 \mathrm{H}$, pyr-H); ${ }^{13} \mathrm{C}-\mathrm{NMR}$ (DMSO-d6): 14.36 (2 $\left.\mathrm{C}, 2 \mathrm{CH}_{3}\right), 41.34\left(2 \mathrm{C}, 2 \mathrm{CH}_{2}\right), 54.18(2 \mathrm{C}, 2 \mathrm{CH}), 55.32(2 \mathrm{C}$, $\left.2 \mathrm{OCH}_{3}\right), 125.65,127.73,128.54,139.42$ (12 C, $\left.2 \mathrm{Ph}-\mathrm{C}\right)$, 113.95, 125.78, 129.94, 162.48 (12 C, 2 Ph-C), 131.76, 140.42, 151.80 (5 C, pyr-C), 167.43 (2 C, 2 CO), 168.35 (2 C, 2 C=N), 176.88 (2 C, 2 CO); MS, m/z (\%): 754 (12) (M) ${ }^{+}$; Anal. Calcd.

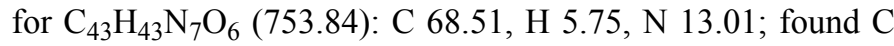
68.40, H 5.70, N 12.90 .

N,N'-Bis[1-(4-chlorophenyl-1-methylhydrazonyl)-2-Lphenylalaninyl]-3,5-(diaminocarbonyl)pyridine (7d): Yield, 64 $\%$; M. p. $234-236^{\circ} \mathrm{C}(\mathrm{AcOH}) ;[\alpha]^{25} \mathrm{D}=-86$ (c=0.5, DMF); IR (KBr) vmax in $\mathrm{cm}^{-1}$ : 3398-3354 (NH), 3085 (CH-Ar), 2978 (CH-aliph.), 1654, 1535, 1252 (CO, amides I-III) $\mathrm{cm}^{-1} ;{ }^{1} \mathrm{H} \mathrm{MR}$ (DMSO-d6, $\delta$ ppm): 1.10 (s, $\left.6 \mathrm{H}, 2 \mathrm{CH}_{3}\right), 3.26$ (d, $4 \mathrm{H}, 2 \mathrm{CH}_{2}$ ), 4.65-4.70 (m, $2 \mathrm{H}, 2 \mathrm{CH}), 7.15-7.72(\mathrm{~m}, 18 \mathrm{H}, 4 \mathrm{Ph}-\mathrm{H}), 8.52$, $8.68\left(2 \mathrm{~s}, 4 \mathrm{H}, 4 \mathrm{NH}, \mathrm{D}_{2} \mathrm{O}\right.$ exchangeable), 8.76, $9.14(2 \mathrm{~s}, 3 \mathrm{H}$, pyr-H). ${ }^{13} \mathrm{C}-\mathrm{NMR}$ (DMSO-d6): $14.52\left(2 \mathrm{C}, 2 \mathrm{CH}_{3}\right), 41.28$ (2 
$\left.\mathrm{C}, 2 \mathrm{CH}_{2}\right), 53.85(2 \mathrm{C}, 2 \mathrm{CH}), 125.64,127.70,128.62,139.38$ (12 C, 2 Ph-C), 128.82, 129.55, 131.65, 136.35 (12 C, 2 Ph-C), 131.80, 140.40, 151.82 (5 C, pyr-C), 167.45 (2 C, 2 CO), $168.48(2 \mathrm{C}, 2 \mathrm{C}=\mathrm{N}), 176.86$ (2 C, $2 \mathrm{CO}) ; \mathrm{MS}, \mathrm{m} / \mathrm{z}(\%): 763$ (32) (M) ${ }^{+}$; Anal. Calcd. for $\mathrm{C}_{41} \mathrm{H}_{37} \mathrm{Cl}_{2} \mathrm{~N}_{7} \mathrm{O}_{4}$ (762.68): $\mathrm{C} 64.57$, $\mathrm{H} 4.89, \mathrm{Cl} 9.30, \mathrm{~N} 12.86$; found $\mathrm{C} 64.50, \mathrm{H} 4.80, \mathrm{Cl} 9.22, \mathrm{~N}$ 12.80 .

\section{$N, N$ '-Bis [1-(4-fluorophenyl-1-methylhydrazonyl)-2-L-}

phenylalaninyl]-3,5-(diaminocarbonyl)pyridine (7e): Yield, 70 \%; M. p. $216-218^{\circ} \mathrm{C}(\mathrm{AcOH}) ;[\alpha]^{25} \mathrm{D}^{=-116}$ (c=0.5, DMF); IR (KBr) vmax in $\mathrm{cm}^{-1}$ : 3378-3342 (NH), 3080 (CH-Ar), 2972 (CH-aliph.), 1652, 1533, 1254 (CO, amides I-III) cm ${ }^{-1} ;{ }^{1} \mathrm{H} \mathrm{MR}$ (DMSO-d6, $\delta$ ppm): 1.15 (s, $6 \mathrm{H}, 2 \mathrm{CH}_{3}$ ), 3.24 (d, $4 \mathrm{H}, 2 \mathrm{CH}_{2}$ ), 4.68-4.72 (m, 2 H, $2 \mathrm{CH}), 7.10-7.75$ (m, $18 \mathrm{H}, 4 \mathrm{Ph}-\mathrm{H}), 8.68$, $8.70\left(2 \mathrm{~s}, 4 \mathrm{H}, 4 \mathrm{NH}, \mathrm{D}_{2} \mathrm{O}\right.$ exchangeable $), 8.75,9.10(2 \mathrm{~s}, 3 \mathrm{H}$, pyr-H); ${ }^{13} \mathrm{C}-\mathrm{NMR}$ (DMSO-d6): $14.56\left(2 \mathrm{C}, 2 \mathrm{CH}_{3}\right), 41.32$ (2 C, $\left.2 \mathrm{CH}_{2}\right), 53.88(2 \mathrm{C}, 2 \mathrm{CH}), 125.58,127.64,128.60,139.44$ (12 C, 2 Ph-C), 114.65, 128.62, 129.68, 164.78 (12 C, 2 Ph-C), 131.76, 140.38, 151.80 (5 C, pyr-C), 167.41 (2 C, 2 CO), $168.52(2 \mathrm{C}, 2 \mathrm{C}=\mathrm{N}), 176.82$ (2 C, 2CO); MS, m/z (\%): 730 (25) $(\mathrm{M})^{+}$; Anal. Calcd. for $\mathrm{C}_{41} \mathrm{H}_{37} \mathrm{~F}_{2} \mathrm{~N}_{7} \mathrm{O}_{4}$ (729.77): C 67.48, H 5.11, N 13.44; found C 67.40, H 5.05, N 13.40.

N,N'-Bis[1-(2-pyridyl-1-methylhydrazonyl)-2-L-

phenylalaninyl]-3,5-(diaminocarbonyl)pyridine (8a): Yield, $65 \%$; M. p. $217-219^{\circ} \mathrm{C}\left(\mathrm{AcOH} / \mathrm{H}_{2} \mathrm{O}\right) ;[\alpha]^{25} \mathrm{D}^{=-} 92(\mathrm{c}=0.5$, $\mathrm{DMF})$; IR (KBr) vmax in $\mathrm{cm}^{-1}: 3454-3324(\mathrm{NH}), 3088(\mathrm{CH}-$ Ar), 2974 (CH-aliph.), 1655, 1534, 1253 (C=O, amides I-III) $\mathrm{cm}^{-1} ;{ }^{1} \mathrm{H}$ MR (DMSO-d6, $\delta$ ppm): $0.99\left(\mathrm{~s}, 6 \mathrm{H}, 2 \mathrm{CH}_{3}\right), 3.62$ (d, $\left.4 \mathrm{H}, 2 \mathrm{CH}_{2}\right), 4.64-4.70(\mathrm{~m}, 2 \mathrm{H}, 2 \mathrm{CH}), 7.02-7.60(\mathrm{~m}, 10 \mathrm{H}$, $2 \mathrm{Ph}-\mathrm{H}), 7.75-8.56$ (m, $6 \mathrm{H}, 2$-pyridyl-H), 8.65, 8.92 (2 s, $4 \mathrm{H}$, $4 \mathrm{NH}, \mathrm{D}_{2} \mathrm{O}$ exchangeable), $8.80(\mathrm{~s}, 2 \mathrm{H}$, pyridyl-H), 8.75, 9.08 (2 s, 3 H, 3,5-pyridyl-H); ${ }^{13} \mathrm{C}-\mathrm{NMR}$ (DMSO-d6): 13.65 (2 C, 2 $\left.\mathrm{CH}_{3}\right), 41.82\left(2 \mathrm{C}, 2 \mathrm{CH}_{2}\right), 53.74(2 \mathrm{C}, 2 \mathrm{CH}), 125.35,127.62$, 128.55, 139.58 (12 C, 2 Ph-C), 122.88, 126.12, 131.68, 135.84, $140.32,148.65,152.28,154.48$ (15C, 3 pyr-C), 144.98 (2 C, 2 $\mathrm{C}=\mathrm{N}), 167.72$ (2 C, $2 \mathrm{CO}), 176.85$ (2 C, $2 \mathrm{CO}) ; \mathrm{MS}, \mathrm{m} / \mathrm{z}(\%)$ : 696 (10) (M) ${ }^{+}$; Anal. Calcd. for $\mathrm{C}_{39} \mathrm{H}_{37} \mathrm{~N}_{9} \mathrm{O}_{4}$ (695.76): C 67.32, H 5.36, N 18.12; found C 67.21, H 5.30, N 18.05.

\section{N,N'-Bis[1-(3-pyridyl-1-methylhydrazonyl)-2-L-}

phenylalaninyl]-3,5-(diaminocarbonyl)pyridine (8b): Yield, 55 \%; M. p. $241-243^{\circ} \mathrm{C}(\mathrm{EtOH}) ;[\alpha]^{25} \mathrm{D}^{=-75}$ (c=0.5, DMF); IR (KBr) vmax in $\mathrm{cm}^{-1}: 3415-3332(\mathrm{NH}), 3075$ (CH-Ar), 2982 (CH-aliph.), 1652, 1534, 1255 (CO, amides I-III) $\mathrm{cm}^{-1} .{ }^{1} \mathrm{H} \mathrm{MR}$ (DMSO-d6, $\delta$ ppm): 1.05 (s, $6 \mathrm{H}, 2 \mathrm{CH}_{3}$ ), 3.75 (d, $4 \mathrm{H}, 2 \mathrm{CH}_{2}$ ), 4.60-4.66 (m, $2 \mathrm{H}, 2 \mathrm{CH}), 7.00-7.58(\mathrm{~m}, 10 \mathrm{H}, 2 \mathrm{Ph}-\mathrm{H})$, 7.70-8.50 (m, $6 \mathrm{H}$, pyridyl-H), 8.65, 8.86 (2 s, $4 \mathrm{H}, 4 \mathrm{NH}, \mathrm{D}_{2} \mathrm{O}$ exchangeable), 8.75, 9.10 (2 s, $3 \mathrm{H}, 3,5$-pyridyl-H), 9.18 (s, 2 $\mathrm{H}$, pyridyl-H-2); ${ }^{13} \mathrm{C}-\mathrm{NMR}$ (DMSO-d6): 12.98 (2 C, $2 \mathrm{CH}_{3}$ ), $41.68\left(2 \mathrm{C}, 2 \mathrm{CH}_{2}\right), 53.84(2 \mathrm{C}, 2 \mathrm{CH}), 125.64,127.58,128.72$, 139.67 (12 C, 2 Ph-C), 122.56, 125.52, 136.92, 150.75, 151.24 (10 C, 3-Pyridyl-C), 131.48, 140.65, 152.32 (5 C, 3,5-pyridylC), 167.05 (2 C, 2 CO), $168.42(2 \mathrm{C}, 2 \mathrm{C}=\mathrm{N}), 176.78(2 \mathrm{C}, 2$ $\mathrm{CO}) ; \mathrm{MS}, \mathrm{m} / \mathrm{z}$ (\%): 696 (35) (M) ${ }^{+}$; Anal. Calcd. for $\mathrm{C}_{39} \mathrm{H}_{37} \mathrm{~N}_{9} \mathrm{O}_{4}$ (695.76): $\mathrm{C}$ 67.32, $\mathrm{H} \mathrm{5.36,} \mathrm{N} \mathrm{18.12;} \mathrm{found} \mathrm{C}$ 67.20, H 5.28, N 18.05.
$N, N$ '-Bis [1-(4-pyridyl-1-methylhydrazonyl)-2-L-

phenylalaninyl]-3,5-(diaminocarbonyl)pyridine (8c): Yield, 62 $\%$; M. p. $255-257^{\circ} \mathrm{C}(\mathrm{MeOH}) ;[\alpha]^{25} \mathrm{D}^{=-102}$ (c=0.5, DMF); IR (KBr) vmax $\mathrm{n} \mathrm{cm}^{-1}$ : 3410-3330 (NH), 3078 (CH-Ar), 2986 (CH-aliph.), 1653, 1532, 1251 (CO, amides I-III) $\mathrm{cm}^{-1} ;{ }^{1} \mathrm{H} \mathrm{MR}$ (DMSO-d6, $\delta$ ppm): 0.98 (s, $\left.6 \mathrm{H}, 2 \mathrm{CH}_{3}\right), 3.78$ (d, $4 \mathrm{H}, 2 \mathrm{CH}_{2}$ ), 4.62-4.65 (m, 2 H, 2 CH), 6.98-7.46 (m, 10 H, 2 Ph-H), 7.78, $8.70\left(\mathrm{~d}, 8 \mathrm{H}\right.$, pyridyl-H) $8.68,8.88\left(2 \mathrm{~s}, 4 \mathrm{H}, 4 \mathrm{NH}, \mathrm{D}_{2} \mathrm{O}\right.$ exchangeable), 8.78, 9.12 (2 s, $3 \mathrm{H}, 3$,5-pyridyl-H); ${ }^{13} \mathrm{C}-\mathrm{NMR}$ (DMSO-d6): $13.12\left(2 \mathrm{C}, 2 \mathrm{CH}_{3}\right), 41.60\left(2 \mathrm{C}, 2 \mathrm{CH}_{2}\right), 53.80(2$ C, $2 \mathrm{CH}$ ), 125.60, 127.50, 128.65, 139.60 (12 C, 2 Ph-C), 123.65 , 136.52, 148.65 (10 C, pyridyl-C), 131.55, 140.32, 152.44 (5 C, 3,5-pyridyl-C), 167.00 (2 C, 2 CO), 168.30 (2 C, $2 \mathrm{C}=\mathrm{N}), 176.84$ (2 C, $2 \mathrm{CO}) ; \mathrm{MS}, \mathrm{m} / \mathrm{z}(\%): 696(16)(\mathrm{M})^{+}$; Anal. Calcd. for $\mathrm{C}_{39} \mathrm{H}_{37} \mathrm{~N}_{9} \mathrm{O}_{4}$ (695.76): calcd. C 67.32, H 5.36, N 18.12; found C 67.18, H 5.24, N 18.08.

Synthesis of compounds 9-11: A mixture of 4 (1 mmol) and dicarboxylic acid anhydride derivatives (phthalic anhydride, tetrachlorophthalic anhydride, 1, 8-naphthalenedicarboxylic acid anhydride or 2, 3-pyridinedicarboxylic acid anhydride, 2 $\mathrm{mmol})$ was refluxed in glacial acetic acid $(50 \mathrm{ml})$ for $6 \mathrm{~h}$. The reaction mixture was poured into ice-water, the obtained precipitate was collected by filtration, washed with water, dried, and crystallized from $\mathrm{DMF} / \mathrm{H}_{2} \mathrm{O}$ to give the corresponding bisimide hexacarboxamide derivatives 9-11, respectively.

No-Dinicotinoyl-bis(L-phenylalaninyl-isoindoline-1,3-dioneimide) (9): Yield, 72\%; M. p. $256-258^{\circ} \mathrm{C}$; $[\alpha]^{25} \mathrm{D}=-117(\mathrm{c}=0.5$, DMF); IR (KBr) vmax in $\mathrm{cm}^{-1}: 3398-3343(\mathrm{NH}), 3078(\mathrm{CH}-$ Ar), 2986 (CH-aliph.), 1653, 1533, 1254 (C=O, amides I-III) $\mathrm{cm}^{-1} ;{ }^{1} \mathrm{H}$ MR (DMSO-d6, $\left.\delta \mathrm{ppm}\right): 3.40$ (d, $4 \mathrm{H}, 2 \mathrm{CH}_{2}$ ), 4.56-4.63 (m, $2 \mathrm{H}, 2 \mathrm{CH}), 7.08-8.05$ (m, $18 \mathrm{H}, 4 \mathrm{Ph}-\mathrm{H}), 8.45$, $9.00\left(2 \mathrm{~s}, 3 \mathrm{H}\right.$, pyr-H), 8.64, $8.75\left(2 \mathrm{~s}, 4 \mathrm{H}, 4 \mathrm{NH}, \mathrm{D}_{2} \mathrm{O}\right.$ exchangeable); ${ }^{13} \mathrm{C}-\mathrm{NMR}$ (DMSO-d6): $40.84\left(2 \mathrm{C}, 2 \mathrm{CH}_{2}\right)$, 53.03 (2 C, 2 CH), 124.34, 127.42, 128.22, 138.86 (12 C, 2 PhC), 127.24, 131.22, 131.86, (12 C, 2 Phth-C), 131.65, 140.14, 152.13 (5 C, pyr-C), 164.56 (4 C, 4 CO-imide), 167.12, 170.15 (4 C, 4 CO-amide); MS, m/z (\%): 750 (14) (M) ${ }^{+}$; Anal. Calcd. for $\mathrm{C}_{41} \mathrm{H}_{31} \mathrm{~N}_{7} \mathrm{O}_{8}$ (749.72): $\mathrm{C}$ 65.68, $\mathrm{H} 4.17, \mathrm{~N} \mathrm{13.08}$; found $\mathrm{C}$ 65.60, H 4.10, N 13.00 .

\section{$N \alpha$-Dinicotinoyl-bis $(L-$}

phenylalaninylbenzo[de]isoquinoline-1,3(2H)-dione-imide) (10): Yield, 65\%; M. p. $264-266^{\circ} \mathrm{C}$; $[\alpha]^{25} \mathrm{D}=-68$ (c=0.5, DMF); IR (KBr) vmax in $\mathrm{cm}^{-1}:$ 3416-3332 (NH), 3073 (CH-Ar), 2987 (CH-aliph.), 1655, 1535, $1255(\mathrm{C}=\mathrm{O}$, amides I-III $) \mathrm{cm}^{-1} ;{ }^{1} \mathrm{H}$ MR (DMSO-d6, $\delta$ ppm): 3.40 (d, 4 H, $2 \mathrm{CH}_{2}$ ), 4.45-4.52 (m, 2 $\mathrm{H}, 2 \mathrm{CH}), 7.12-7.86$ (m, $22 \mathrm{H}, \mathrm{Ar}-\mathrm{H}), 8.57,9.02$ (2 s, $3 \mathrm{H}$, pyr$\mathrm{H}), 8.60,8.78\left(2 \mathrm{~s}, 4 \mathrm{H}, 4 \mathrm{NH}\right.$, exchangeable with $\left.\mathrm{D}_{2} \mathrm{O}\right) ;{ }^{13} \mathrm{C}$ NMR (DMSO-d6): 41.45 (2 C, $\left.2 \mathrm{CH}_{2}\right), 53.12(2 \mathrm{C}, 2 \mathrm{CH})$, $122.12,124.65,127.95,130.10,136.86,137.35(20 \mathrm{C}$, naphthyl-C), 125.60, 127.54, 128.54, 139.68 (12 C, 2 Ph-C), 131.62, 140.18, 152.17 (5 C, pyr-C), 158.55 (4 C, 4 COimide), 167.24, 170.26 (4 C, 4 CO-amide); $\mathrm{MS}, \mathrm{m} / \mathrm{z}$ (\%): 850 (8) (M) ${ }^{+}$; Anal. Calcd. for $\mathrm{C}_{49} \mathrm{H}_{35} \mathrm{~N}_{7} \mathrm{O}_{8}$ (849.84): $\mathrm{C} 69.25, \mathrm{H}$ 4.15, N 11.54; found C 69.12, H 4.05, N 11.43. 
Na-Dinicotinoyl-bis[L-phenylalaninylpyrrolo[3,4b]pyridine-5,7-dione-imide] (11): Yield, 68\%; M. p. $234-236^{\circ} \mathrm{C} ;[\alpha]^{25} \mathrm{D}^{=-96}(\mathrm{c}=0.5, \mathrm{DMF})$; IR $(\mathrm{KBr}) v \max$ in $\mathrm{cm}^{-1}$ : 3445-3313 (NH), 3083 (CH-Ar), 2992 (CH-aliph.), 1653, 1535, $1252\left(\mathrm{C}=\mathrm{O}\right.$, amides I-III) $\mathrm{cm}^{-1} ;{ }^{1} \mathrm{H}$ MR (DMSO-d6, $\delta$ ppm): $3.45\left(\mathrm{~d}, 4 \mathrm{H}, 2 \mathrm{CH}_{2}\right), 4.50-4.61(\mathrm{~m}, 2 \mathrm{H}, 2 \mathrm{CH})$, 6.94-7.48 (m, $10 \mathrm{H}, 2 \mathrm{Ph}-\mathrm{H}), 8.62,9.05(2 \mathrm{~s}, 3 \mathrm{H}$, pyr-H), 7.96-8.32 (m, 4 H, pyr-H), 8.95 (t, 2 H, pyr-H), 8.68, 8.75 (2 s, $4 \mathrm{H}, 4 \mathrm{NH}$, exchangeable with $\mathrm{D}_{2} \mathrm{O}$ ); ${ }^{13} \mathrm{C}-\mathrm{NMR}$ (DMSO-d6): $42.28\left(2 \mathrm{C}, \mathrm{CH}_{2}\right), 52.87(2 \mathrm{C}, 2 \mathrm{CH}), 124.32,128.36,129.43$, 138.76 (12 C, 2 Ph-C), 127.21, 128.05, 131.66, 137.94, 140.12, $145.12,152.10,152.56$ (15 C, pyr-C), 164.63, 164.95 (4 C, 4 CO-Imide), 169.48, 170.34 (4 C, 4 CO-amide); MS, m/z (\%): 752 (20) (M) ${ }^{+}$; Anal. Calcd. for $\mathrm{C}_{39} \mathrm{H}_{29} \mathrm{~N}_{9} \mathrm{O}_{8}$ (751.70): C 62.31, H 3.89, N 16.77; found C 62.20, H 3.80, N 16.70.

\section{Antimicrobial activity}

The antimicrobial activities of the synthesized compounds were determined by the agar diffusion method as recommended by the National Committee for Clinical Laboratory Standards (NCCLS) [25]. The concentrations of the tested compounds $(10 \mu \mathrm{g} / \mathrm{ml})$ were used according to modified Kirby-Bauer's disk diffusion method [26]. The degree of inhibition is measured in comparison with that of Chloramphenicol ${ }^{\circledR}$ and fusidic acid taken as standards.

\section{Conclusion}

In the present work, a series of tetracarboxamide and macrocyclic tripeptides has been prepared starting from 3,5bis[N-(1-hydrazinyl-1-oxo-3-phenylpropan-2-yl)]pyridinecarboxamide as starting material. Some of the synthesized compounds were screened as antimicrobial agents.

\section{Acknowledgment}

The authors extend their appreciation to the Deanship of Scientific Research at Tibah University for support the work through the research group project No. 1436/6850.

\section{References}

1. Padhi A, Sengupta M, Sengupta S, Roehm K H, Sonawane A. Antimicrobial peptides and proteins in mycobacterial therapy: current status and future prospects. Tuberculosis 2014; 94: 363-373.

2. Giordano C, Marchio M, Timofeeva E, Biagini G. Neuroactive peptides as putative mediators of antiepileptic ketogenic diets. Front Neurol 2014; 5: 63-67.

3. Robinson SD, Safavi-Hemami H, McIntosh LD, Purcell AW, Norton RS, Papenfuss AT. Diversity of conotoxin gene superfamilies in the venomous snail, Conus victoriae. PLoS One 2014; 9: 87640-87648.

4. Mamolo MG, Zampieri D, Falagiani V, Vio L, Fermeglia M, Ferrone M, Pricl S, Banfi E, Scialino G. Antimycobacterial activity of new 3-substituted 5(pyridin-4-yl)-3H-1,3,4-oxadiazol-2-one and 2-thione derivatives. Preliminary molecular modeling investigations. ARKIVOC 2004; 5: 231-250.

5. Prachayasittikul S, Pingaew R, Worachartcheewan A, Sinthupoom N, Prachayasittikul V, Ruchirawat S, Prachayasittiku V. Roles of pyridine and pyrimidine derivatives as privileged scaffolds in anticancer agents. Mini Rev Med Chem 2017; 17: 869-901.

6. Amorim R, de Meneses MDF, Borges JC, da Silva Pinheiro LC, Caldas LA, Cirne-Santos CC, de Mello MVP, de Souza AMT, Castro HC, de Palmer Paixao ICN, Campos RM, Bergmann IE, Malirat V, Bernardino AMR, Rebello MA, Ferreira DF. Thieno[2,3-b]pyridine derivatives: a new class of antiviral drugs against Mayaro virus. Arch Virol 2017; 162: 1577-1587.

7. Liu Z, Tian Y, Liu J, Huang B, Kang D, Clercq E, Daelemans D, Pannecouque C, Zhan P, Liu X. Design, synthesis and anti-HIV evaluation of novel diarylpyridine derivatives as potent HIV-1 NNRTIs. Eur J Med Chem 2017; 140: 383-391.

8. LiuW, Timothy J, Jensen Frank R, Fronczek Robert P, Kevin M, Smith Graca M, Vicente H. Synthesis and cellular studies of nonaggregated water-soluble phthalocyanines. J Med Chem 2005; 48: 1033-1041.

9. TuckerTJ, Sisko JT, Tynebor RM, Williams TM, Felock PJ, Flynn JA, Lai M, Liang Y, McGaughey G, Liu M, Miller M, Moyer G, Munshi V, Poehnelt RP, Prasad S, Reid JC, Sanchez R, Torrent M, Vacca JP, Wan BL, YanY. Discovery of $3-\{5-[(6-a m i n o-1 H-$ pyrazolo[3,4-b] pyridine-3-yl) methoxy] -2-chlorophenoxy 3 -5chlorobenzonitrile (MK-4965): a potent, orally bioavailable HIV-1 non- nucleoside reverse transcriptase inhibitor with improved potency against key mutant viruses. J Med Chem 2008; 51: 6503-6511.

10. Zhu Q, Wang X, Chu Z, He G, Dong G, Xu Y. Design, synthesis and biological evaluation of novel imidazo[4,5c]pyridinecarboxamide derivatives as PARP-1 inhibitors. Bioorg Med Chem Lett 2013; 23: 1993-1996.

11. Klimesova V, Herzigova P, Palat K, Machaacek M, Stolarikova J, Dahse HM, Möllmann U. The synthesis and antimycobacterial properties of 4-(substituted benzylsulfanyl)pyridine-2-carboxamides. ARKIVOC 2012; 3: 90-103.

12. Tabrizi MA, Baraldi PG, Saponaro G, Moorman AR, Romagnoli R, Preti D, Baraldi S, Corciulo C, Vincenzi F, Borea PA, Varani K. Design, synthesis, and pharmacological properties of new heteroarylpyridine/ heteroarylpyrimidine derivatives as $\mathrm{CB}(2)$ cannabinoid receptor partial agonists. J Med Chem 2013; 56: 1098-1112.

13. Fukumizu A, Tsuda Y, Wanaka K, Tada M, Okamoto S, Hijikata-Okunomiya A, OkadaY. Amino acids and peptides. LIII. Synthesis and biological activities of some pseudo-peptide analogs of PKSI-527, a plasma kallikrein selective inhibitor: the importance of the peptide backbone. Chem Pharm Bull 1999; 47: 1141-1144. 
14. Stanchev M, Tabakova S, Videnov G, Golovinsky E, Jung G. Synthesis and antimicrobial activity in vitro of new amino acids and peptides containing thiazole and oxazole moieties. Arch Pharm 1999; 332: 297-304.

15. Nishitani S, Ijichi C, Takehana K, Fujitani S, Sonaka I. Pharmacological activities of branched-chain amino acids: specificity of tissue and signal transduction. Biochem Biophys Res Commun 2004; 313: 387-389.

16. Ibrahim AA, Mohamed AM, Amr AE, Al-Omar MA. Synthesis of chiral linear and macrocyclic candidates: II. Synthesis and investigation of 3, 5-bis-linear and macrocyclic tetrapeptide Schiff base pyridine derivatives. Russ J Gen Chem 2015; 85: 1506-1512.

17. Azab ME, Amr AE. Synthesis of chiral linear and macrocyclic candidates: III. Synthesis and antimicrobial activity of linear tetrapeptide and macrocyclic pentapeptide Schiff Bases. Russ J Gene Chem 2015; 85: 1513-1521.

18. Amr AE, Abd El-Salam OI, Al-Omar MA. Synthesis of chiral macrocycles: I. Synthesis and study of cyclo( $\mathrm{N \alpha}$ dinicotinoyl)pentapeptide candidates. Russ J Gen Chem 2015; 85: 1161-1166.

19. Azab ME, Amr AE, Al-Omar MA. Synthesis of chiral macrocyclic candidates: IV. Synthesis and antimicrobial activity of some tricyclooctacosa(triaconta)hexaene bisSchiff base derivatives. Russ J Gen Chem 2015; 85: 1952-1958.

20. Amr AE. Synthesis of some new linear and chiral macrocyclic pyridine carbazides as analgesic and anticonvulsant agents. Z Naturforsch 2005; 60: 990-998.

21. Abu-Ghalia MH, Abd El-Hamid M, Zweel MA, Amr AE, Moafi S A. Synthesis and reactions of new chiral linear and macrocyclic tetra and Penta-peptide candidates. $\mathrm{Z}$ Naturforsch 2012; 67: 806-818.
22. Amr AE, Abo-Ghalia MH, Abdalla MM. Synthesis of novel macrocyclic peptidocalix[4]arenes and peptidopyridines as precursors for potential molecular metallacages, chemo-sensors and biologically active candidates. Z Naturforsch 2006; 61: 1335-1345.

23. Amr AE, Abou-Ghalia MH, Abdallah MM. Synthesis of new (No-dipicolinoyl)-bis-L-valyl-L-phenylalanyl linear and macrocyclic bridged peptides as anti-inflammatory agents. Arch Pharm Chem Life Sci 2007; 340: 304-309.

24. Khayyat S, Amr AE, Abd El-Salam OI, Al-Omar MA, Mohamed MA. Analgesic and anti-inflammatory activities of some newly synthesized 3,5bis[(peptidohydrazinyl)pyridine Schiff bases. Int $\mathrm{J}$ Pharmacol 2015; 11: 423-431.

25. Furtada GL, Medeiros AA. Single-disk diffusion testing (Kirby-Bauer) of susceptibility of Proteus mirabilis to chloramphenicol: significance of the intermediate category. J Clin Microbiol 1980; 12: 550-553.

26. Jones RN, Ballow CH, Biedenbach DJ. Multi-laboratory assessment of the linezolid spectrum of activity using the Kirby-Bauer disk diffusion method: Report of the Zyvox Antimicrobial Potency Study (ZAPS) in the United States. Diagn Microbiol Infect Dis 2001; 40: 59-66.

\section{*Correspondence to}

Eman M. Flefel

Department of Chemistry

College of Science

Taibah University

Saudi Arabia 